\title{
Interference Rejection Capability of a Switched Radiometer
}

\author{
Roger E. Clapp
}

\author{
AirTechnology Corporation, Waltham, Mass.
}

(Received May 3, 1965)

\begin{abstract}
The increasing congestion of the radio-frequency spectrum makes necessary a reexamination of current techniques used in radiometric receivers for radio astronomy. Square-wave switching, as currently used, modulates incoming $\mathrm{CW}$ signals and introduces sidebands which can intrude on the presently available windows in the spectrum. This effect can be treated as an apparent deterioration of an ideal square-sided filter. Examples are given, for different filter bandwidths and chopping frequencies. Where radiometry is interference-limited, sinusoidal chopping or a correlation technique avoiding chopping is strongly recommended.
\end{abstract}

\section{Introduction}

Radio astronomy in the high-frequency region, 3 to $30 \mathrm{Mc} / \mathrm{s}$, is becoming increasingly limited by manmade interference. In the next few years this situation will get worse, partly because of the steady increase in the number of transmitting stations and in their power levels, but also as a result of the increase in solar activity as the solar maximum is approached. The intensified solar activity results in increased electron densities in the ionosphere, and in increased signal strengths for the radio signals received at a particular location from the more distant radio transmitters.

At a particular site, the spectral windows available for radio astronomy will tend to dwindle in number and shrink in width. To some extent, the use of steep-sided filters in radio-astronomy receivers will permit the utilization of the spectral windows that remain clear at a specific site. However, there is a basic limitation on the steepness of the filtering action that is imposed by the form of the receivers that are used in most radio-astronomy installations.

Most radio-astronomy receivers use square-wave switching or chopping to make the output as insensitive as possible to variations in receiver gain. The received signal is compared with a standard noise source, and a synchronous detector gives an output which measures the difference between the power level of the received signal and the power level of the standard noise generator. In one form of receiver, the comparison signal is recorded directly. In an alternative form of receiver, the comparison signal is used to control a feedback loop that readjusts the standard noise source to make it match the level of the input signal from the radio-astronomy antenna.

It is the purpose of this article to point out that the switching action has an undesired effect, since it results in a broadening of the spectrum of the interfer- ence signals that are received by the antenna. As a result of the broadening, an interference signal, which is initially outside the band of frequencies that is accepted by the receiver, can be altered to produce spectral components which are contained within the acceptance band of the receiver. This spectral broadening is found to set a limit on the effectiveness of any band-pass filters which may be incorporated into a switched radio-astronomy receiver.

\section{Spectral Broadening Produced by Switching}

The chopping action of a radiometer switch is equivalent to a square-wave modulation. Its effect upon an incident $\mathrm{CW}$ signal can be computed in a straightforward manner. The incident-signal spectrum, which is a single line, is transformed into a broadened spectrum which contains this line but also contains additional lines on either side of this central line. The additional lines are separated from each other by frequency intervals equal to twice the chopping frequency. The total power in the additional lines is equal to the power remaining in the central line, which in turn is equal to one-fourth of the incident power.

The square-wave switching action can be represented mathematically by the Fourier expansion:

$$
g(t)=\frac{1}{2}+\frac{2}{\pi}\left(\cos \beta t-\frac{1}{3} \cos 3 \beta t+\frac{1}{5} \cos 5 \beta t-. .\right) .
$$

The incident $\mathrm{CW}$ signal can be represented by

$$
f(t)=E \cos \alpha t,
$$

where $E$ is the field strength or the voltage level. 
The incident power level is proportional to $\frac{1}{2} E^{2}$. The chopped-CW signal is then given by the product of (1) and (2):

$f(t) g(t)=\frac{E}{2} \cos \alpha t$

$$
\begin{gathered}
+\frac{E}{\pi}\{[\cos (\alpha+\beta) t+\cos (\alpha-\beta) t] \\
-\frac{1}{3}[\cos (\alpha+3 \beta) t+\cos (\alpha-3 \beta) t] \\
+\frac{1}{5}[\cos (\alpha+5 \beta) t+\cos (\alpha-5 \beta) t] \\
\left.-\frac{1}{7}[\cos (\alpha+7 \beta) t+\cos (\alpha-7 \beta) t]+. .\right\} .
\end{gathered}
$$

The power level remaining in the central line is now proportional to $\frac{1}{8} E^{2}$. The power level in the line which

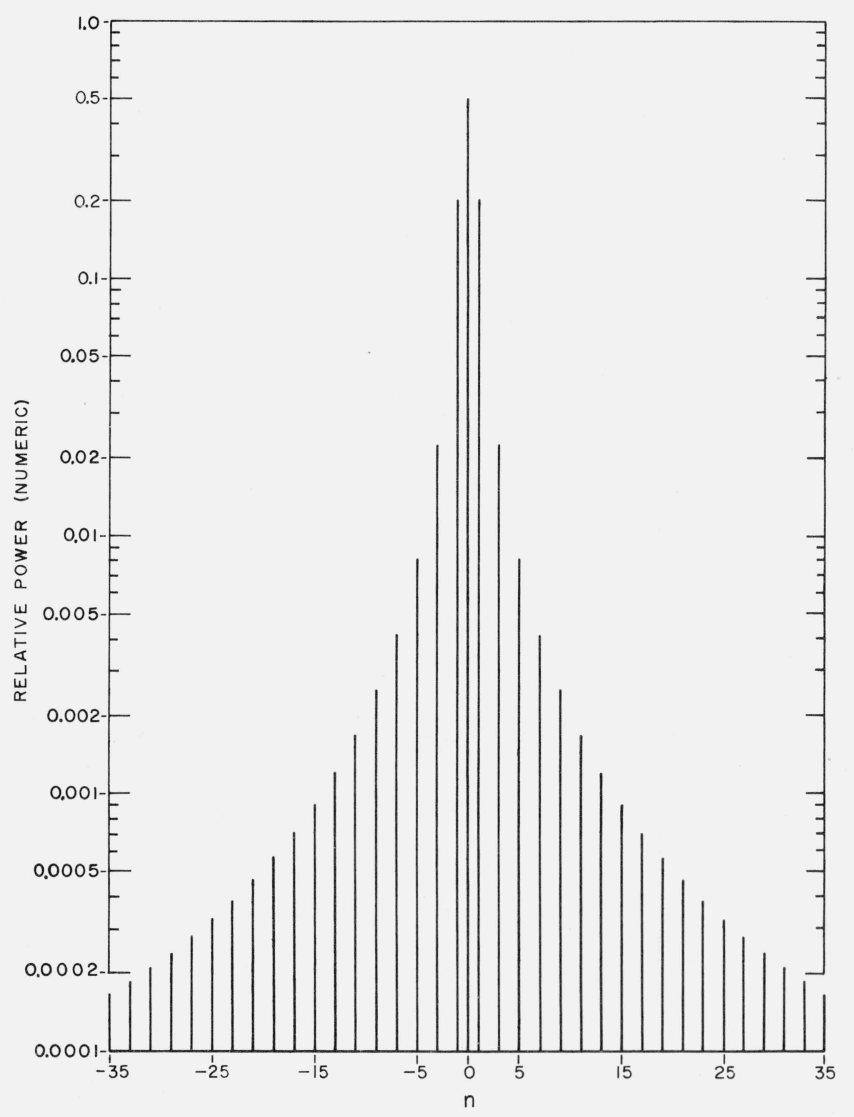

Figure 1. Power spectrum of chopped-CW signal.

The ordinate is the relative power associated with each line in the line spectrum. The frequency of a line in the spectrum is given by $(\alpha+n \beta)$, where $\alpha$ is the frequency of the incident $\mathrm{CW}$ signal and $\beta$ is the chopping frequency. The abscissa is $n$, which takes the incident $C W$ signal and $\beta$ is the choppin
values $0, \pm 1, \pm 3, \pm 5$, and so forth. has the frequency $(\alpha+n \beta)$ is proportional to $\frac{1}{2 \pi^{2} n^{2}} E^{2}$, and is equal to the power level in the line at $(\alpha-n \beta)$.

Figure 1 shows the power spectrum of the chopped. CW signal. The normalization has been chosen to make the total power in the spectrum equal to unity. Thus the power level for the central line is one-half, and the power levels for all of the additional lines add to one-half. While more than 90 percent of the power is contained in the three central lines of the spectrum, it can be seen from figure 1 that a substantial spreading of the remaining 10 percent into frequencies on either side of the three central lines exists.

\section{Selectivity Function for Ideal Filter}

An ideal band-pass filter can be represented by the dotted, square-sided curve which has been labeled (a) in figure 2. The bandwidth that has been chosen for this figure is $10 \mathrm{kc} / \mathrm{s}$, a characteristic bandwidth for radiometry in the crowded $10-\mathrm{Mc} / \mathrm{s}$ region of the electromagnetic spectrum. The curve labeled (b) in figure 2 is the selectivity function which is associated with the ideal filter (a) when this filter is incorporated into a receiving system using square-wave switching at a chopping frequency of $500 \mathrm{c} / \mathrm{s}$. The amplitude of curve (b), at a particular frequency, $f_{1}$, is obtained from the addition of the power levels for those lines in the spectrum of figure 1 which fall within curve (a) when the central line in this spectrum is located at the frequency $f_{1}$. The steps in the selectivity function show the positions of the interfering CW signal for which individual lines in the broadened spectrum move into and out of the filter band. For the 500-c/s chopping frequency used in this example, the steps are all $1 \mathrm{kc} / \mathrm{s}$ wide, except for the $0.5 \mathrm{kc} / \mathrm{s}$ steps where curve (b) crosses curve (a).

The effect of a reduction in the chopping frequency, with no change in the filter bandwidth, can be seen from a comparison of figure 2 with figure 3 . In figure 3 the same $10-\mathrm{kc} / \mathrm{s}$ ideal filter is shown as curve (a), but curve (b) now represents the selectivity function for a chopping frequency of $100 \mathrm{c} / \mathrm{s}$. To simplify the appearance and the graphical construction, curve (b) has been smoothed. This removes the steps, which in this case would have been much finer, only onefifth the width of the steps shown in figure 2 .

The effect of a reduction in the filter bandwidth, with no change in the chopping frequency, can be seen from a comparison of figure 3 with figure 4 . The same chopping frequency of $100 \mathrm{c} / \mathrm{s}$ is used in the calculation of both selectivity functions, but the idealfilter bandwidth of $10 \mathrm{kc} / \mathrm{s}$ in figure 3 is replaced by a bandwidth of $2 \mathrm{kc} / \mathrm{s}$ in the construction of the curves of figure 4 . It can be seen that there is a progressive narrowing of the selectivity functions, in the progression from figure 2 to figure 3 to figure 4 , demonstrating the advantage of the reduction of chopping frequency and the reduction of filter bandwidth, in the effort to remove $\mathrm{CW}$ interference. 


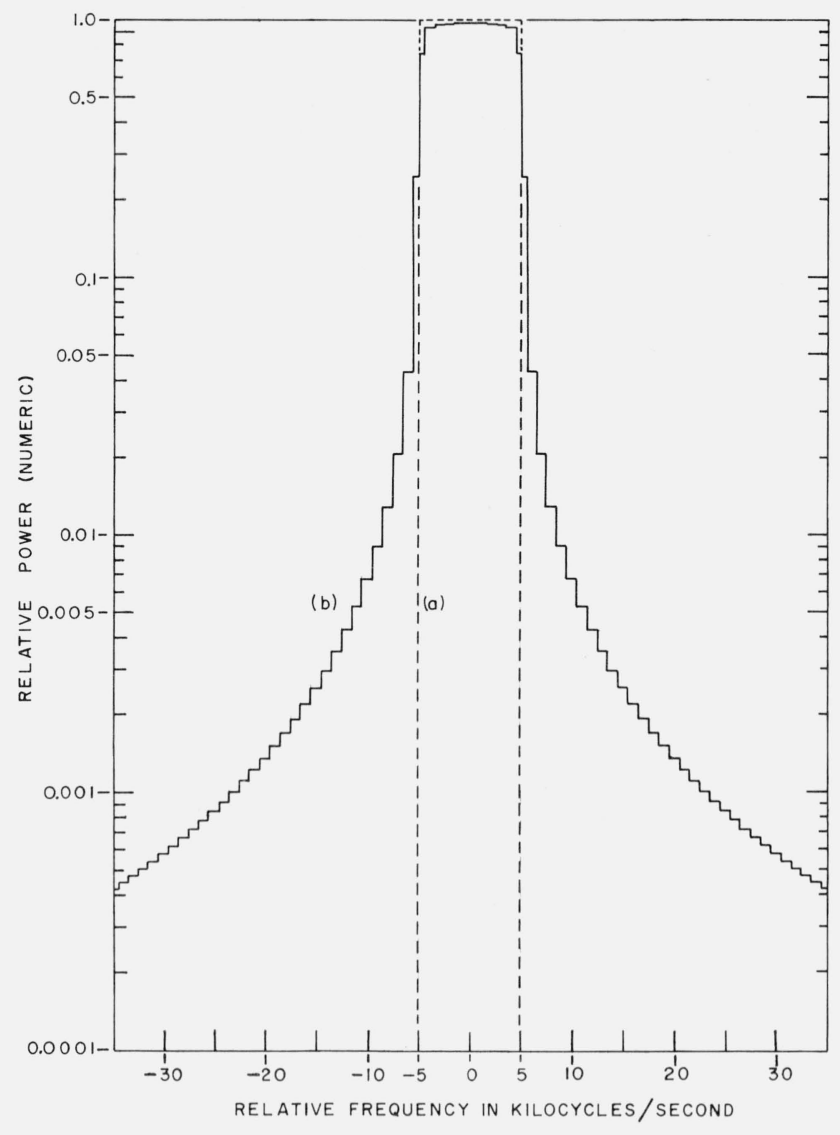

FIGURE 2. Ideal $10-k c / s$ band-pass filter [curve (a), dotted], compared with the selectivity function [curve $(b)$, solid] which results from the use of this ideal filter in a radiometric receiving system using square-wave switching at a chopping frequency of $500 \mathrm{c} / \mathrm{s}$.

Unfortunately, neither the chopping frequency nor the filter bandwidth can be reduced indefinitely, in a practical radio-astronomy installation. Reduction of the filter bandwidth reduces the information input to the system, and requires lengthened integration times to produce a particular precision in the desired signal measurement. Reduction of the chopping frequency is also limited in most cases, since the signal of interest is changing, due either to its intrinsic variation (for example, solar radio emissions) or to the scanning of the antenna beam across the celestial source regions. The chopping period should be kept short in comparison with the integration time, and this in turn should be kept short in comparison with the time for a significant change in the desired radiometric input signal.

For frequencies well outside the pass bands of these ideal filters, the selectivity improvement obtained by a reduction in chopping frequency is directly proportional to the reduction factor. In particular, the reduction in chopping frequency by a factor of five results in an improvement in the selectivity function by $6.99 \mathrm{~dB}$. Similarly, a narrowing in filter bandwidth by a factor of five produces a $6.99 \mathrm{~dB}$ improvement.

In figure 5 the outer wings are shown, for the three selectivity functions whose inner regions are given

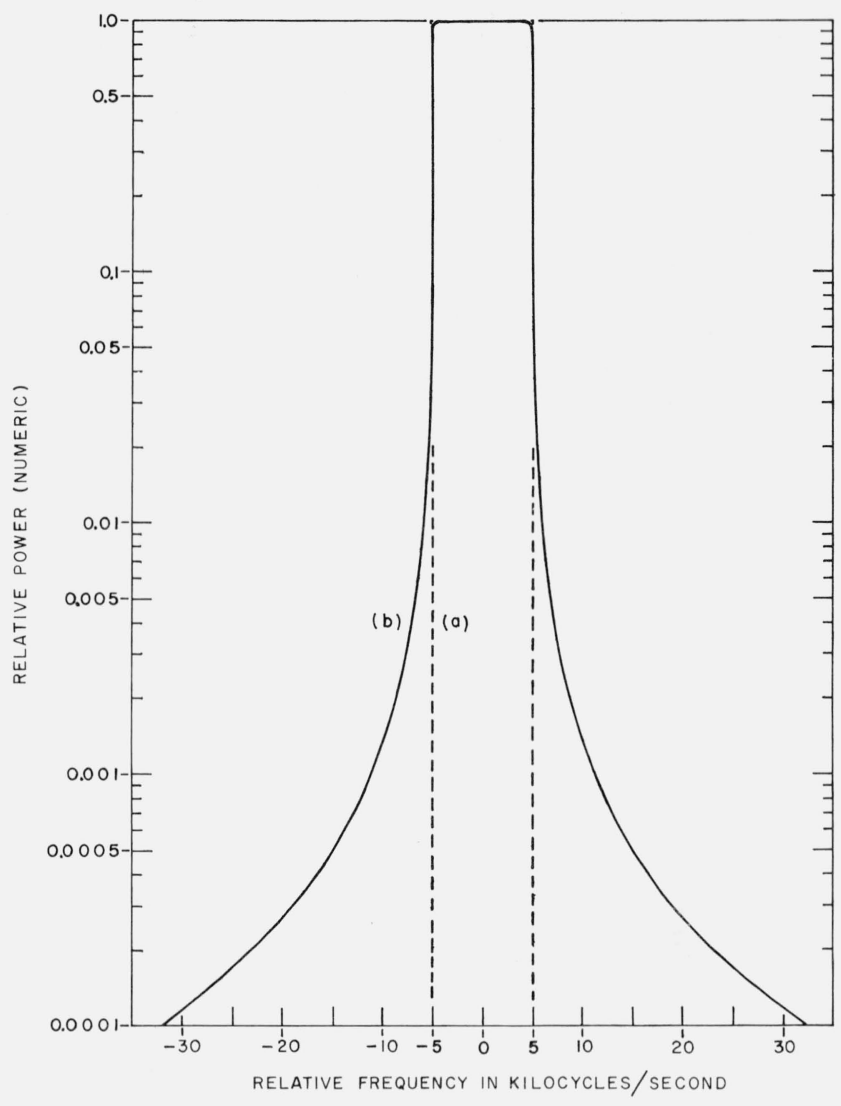

FIgURE 3. Curve (a), ideal 10-kc/s band-pass filter characteristic curve. Curve (b), selectivity function which results when (a) is used in a system with square-wave switching at $100 \mathrm{c} / \mathrm{s}$.

Curve (b) has been smoothed to remove the fine steps.

in figures 2 through 4 . The separation of $6.99 \mathrm{~dB}$ between curves (a) and (b), and between curves (b) and (c), is maintained over all of the wide frequency range in the figure, except for the region very close to the pass bands of the ideal filters. The outer regions of all three curves can be approximated by the formula,

$$
\text { selectivity } \doteq \frac{1}{\pi^{2}} \frac{f_{\mathrm{ch}} f_{\mathrm{bw}}}{\left(f-f_{0}\right)^{2}}
$$

where

$$
\begin{aligned}
f_{\mathrm{ch}} & =\text { chopping frequency, } \\
f_{\mathrm{bw}} & =\text { bandwidth of ideal filter, } \\
f_{0} & =\text { center frequency of filter band, } \\
f & =\text { signal frequency of } \mathrm{CW} \text { interference. }
\end{aligned}
$$

For a spectral region where manmade interference is very strong, the wings shown in figure 5 can be very serious in masking the radio-astronomical radiation which the radiometer is designed to receive. 


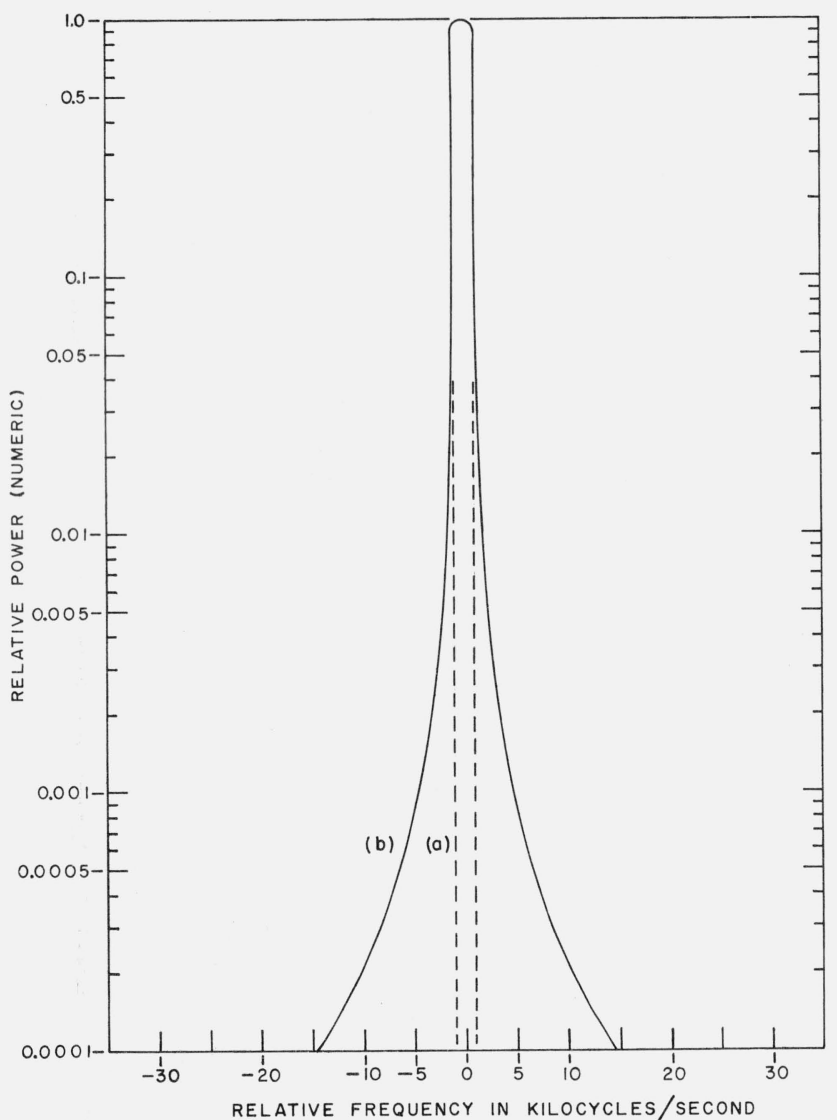

Figure 4. Curve (a), ideal 2-kc/s band-pass filter. Curve (b), selectivity function for square-wave switching at $100 \mathrm{c} / \mathrm{s}$.
It should be noted that the presence of the factor $f_{\mathrm{bw}}$ in the numerator of (4) means that increasing the filter bandwidth, as is done in radiometers to achieve greater differential sensitivity through increased timebandwidth product, has the undesired effect of also increasing the background interference level in proportion to $f_{\mathrm{bw}}$. Figure 6 shows the outer portions of two selectivity functions associated with an ideal filter whose bandwidth is $500 \mathrm{kc} / \mathrm{s}$. The ideal filter characteristic is shown dotted, as curve (a). The two chopping frequencies used in computing the selectivity functions are $1000 \mathrm{c} / \mathrm{s}$, for curve (b), and $100 \mathrm{c} / \mathrm{s}$. for curve (c). As can be seen, the deterioration produced by square-wave switching is not negligible, even though the chopping frequencies are very much smaller than the filter bandwidth.

The discussion thus far has been directed to switched radiometers in which the square-wave switching is between two inputs, one of them the output of a receiving antenna, and the other, a reference noise source. However, the generalization to a phaseswitched receiving system can readily be made. Phase switching is used in Mills Cross and Mills Tee radio-astronomy systems. The signals from two antenna arrays are to be correlated. One array is oriented at right angles to the other array, so that there are two fan beams, and the intersection region is the region of interest. One output is fed to a phase switch, which provides an alternation between a $0^{\circ}$ phase shift and a $180^{\circ}$ phase shift. The other of the two array outputs is then added to the phase-switched output from the first array, and the sum signal is amplified, detected, then synchronously demodulated to give a final output signal which is a measure of the correlation between the signal from one array and the signal from the other array.

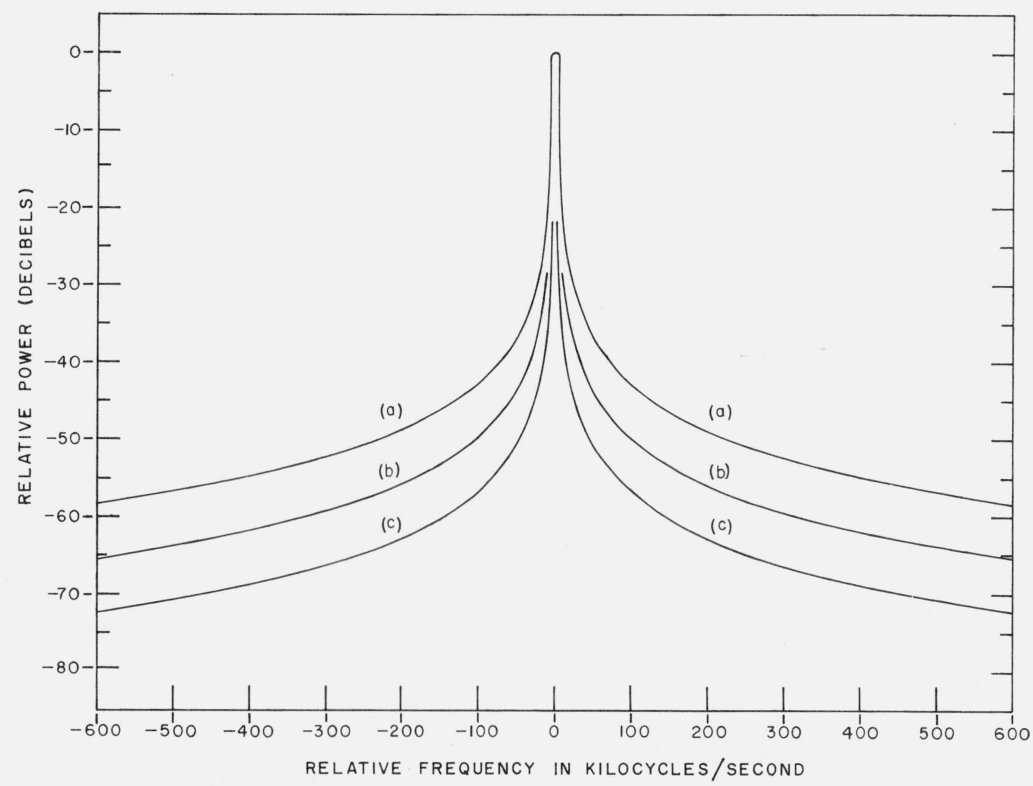

FIGURE 5. The outer portions of the three selectivity functions, expressed in decibels. Curve (a), 10-kc/s filter bandwidth, chopping frequency $500 \mathrm{c} / \mathrm{s}$. Curve (b), 10-kc/s filter bandwidth, chopping frequency $100 \mathrm{c} / \mathrm{s}$. Curve (c), $2-\mathrm{kc} / \mathrm{s}$ filter bandwidth, chopping frequency $100 \mathrm{c} / \mathrm{s}$. 


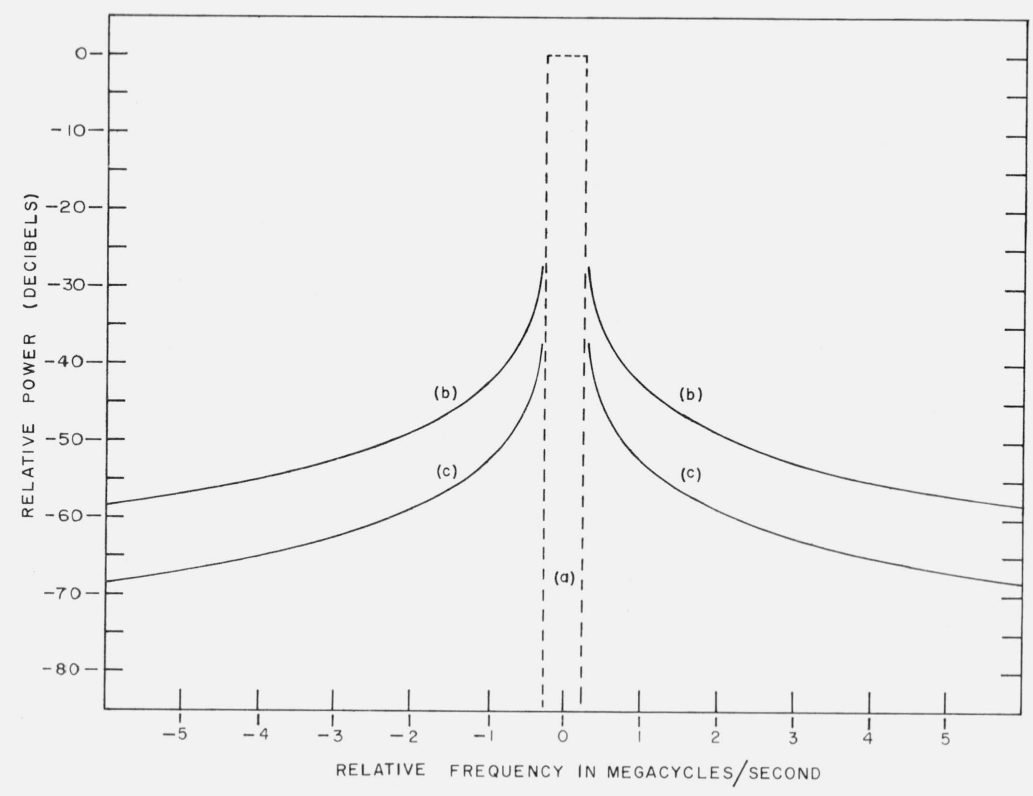

Figure 6. Curve (a), ideal filter characteristic for 500-kc/s bandwidth. Curve (b), selectivity function, for chopping frequency of $1000 \mathrm{c} / \mathrm{s}$. Curve (c), selectivity function, for chopping frequency of $100 \mathrm{c} / \mathrm{s}$.

When an interfering $\mathbf{C W}$ signal is present, the unswitched array passes this signal unmodulated. The phase-switched array, however, passes this signal with a square-wave modulation that can be expressed by the formula:

$$
\bar{g}(t)=\frac{4}{\pi}\left(\cos \beta t-\frac{1}{3} \cos 3 \beta t+\frac{1}{5} \cos 5 \beta t-\ldots\right) .
$$

The effect of this modulation is to introduce all of the lines in the spectrum shown in figure 1, except the central line. When the two signals are thereupon added together, the spectrum of the sum signal contains both the central line and the side-lobe lines, in exactly the proportions shown in figure 1. Consequently, the interference contribution introduced by an interfering CW transmitting station has the same power level in a phase-switched radiometer as in a radiometer using square-wave chopping, for an interfering CW signal which is received on both antenna beams.

It will often happen, however, that interference is received strongly on one antenna beam, but only weakly on the other. In this case, the $180^{\circ}$ phase switch should be connected to the antenna receiving the least interference. This procedure should minimize the background interference level in the radiometer. An interference signal which is received on only one of the two fan beams cannot contribute significantly to the average output of the receiver, since it is not correlated with anything received on the other fan beam. However, it can contribute to the uncorrelated background which accentuates the natural fluctuations associated with the noiselike character of the desired radio-astronomical signals.

\section{Conclusions}

For those portions of the radio-frequency spectrum which contain manmade interference of high power level, a switched radiometer is of limited usefulness, even when steep-sided filters are used in an effort to remove interference at frequencies on either side of a clear "window" in the spectrum. The squarewave switching process is itself a modulation, and it produces sidebands on both sides of the frequency of an incoming interfering signal. Even a perfect receiving system, with an ideal square-sided filter, cannot avoid the reception of these sidebands.

For those portions of the radio-frequency spectrum in which radiometry is (or soon will become) interference-limited, consideration should be given to radiometer designs which avoid square-wave switching. Sinusoidal chopping does not broaden the spectrum of an interfering signal significantly, and is therefore greatly to be preferred over square-wave chopping. Instead of the broad spectrum of figure 1 , there will be only three lines in the spectrum of a sinusoidally chopped CW signal, spaced as are the central three lines in figure 1. A radiometric receiving system using sinusoidal chopping has been described by Clapp [1962]. Radiometric receivers which avoid chopping altogether through the use of hybrid junctions and correlating detectors are particularly insensitive to interference [Clapp and Maxwell, 1966].

\section{References}

Clapp, R. E. (1962), Receiving Apparatus for Radio Frequency Signals, United States Patent No. 3,017,505.

Clapp, R. E., and J. C. Maxwell (1966), Complex-Correlation Radiometer (in preparation).

(Paper 69D11-575) 